\title{
Politische Wettkämpfe?!
}

Die Olympischen Winterspiele 2014 im Spiegel der Presse und der Bevölkerung. Von Regina Greck

\begin{abstract}
Bereits einige Monate vor der Eröffnung der XXII. Olympischen Winterspiele in Sotschi 2014 stand Russland als Gastgeberland in der Kritik. Vor allem die Missachtung von Menschenrechten empörte die deutschen Medien, sodass die politischen Vorwürfe in der Öffentlichkeit den olympischen Sportgedanken zu überlagern schienen. Die vorliegende Studie prüft diesen Eindruck aus zwei Perspektiven, die im Agenda-Setting-Modell verknüpft werden: Sie untersucht überregionale Printmedien hinsichtlich ihrer politischen Themenschwerpunkte in der Berichterstattung über die Spiele, und fragt die Rezipient_innen, welche Aspekte sie wahrgenommen haben. Im Vergleich zeigen sich einige Überschneidungen in der Themenrangfolge der Printmedien und des Publikums.
\end{abstract}

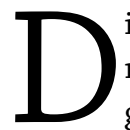
ie XXII. Olympischen Winterspiele 2014 waren kaum nach Sotschi vergeben, schon stand Russland als frisch gekürtes Gastgeberland in der Kritik. Die deutsche Medienlandschaft diskutierte u.a. den enormen finanziellen Aufwand und es regten sich öffentliche Zweifel an der Eignung des Badeortes als Wintersportzentrum. Doch nicht nur die Organisation der Spiele stand im Fokus der Öffentlichkeit, auch die politische Lage des Gastgeberlandes Russland rückte in den Blick. Dabei dominierten u.a. Fragen der Menschenrechte, der Umgang mit politischen Oppositionellen sowie die Diskriminierung Homosexueller die journalistische Berichterstattung. Es entstand der Eindruck, dass der olympische Sportgedanke schon vor Beginn der Spiele in der medialen Diskussion zusehends in den Hintergrund geriet und (sport)politische Aspekte die geplanten Wettkämpfe überlagerten.

Die vorliegende Studie betrachtet diese Hypothese aus zwei Blickwinkeln: Sie untersucht die journalistische Berichterstattung überregionaler deutscher Printtitel hinsichtlich ihrer Themenschwerpunkte und Akteure sowie die Themenwahrnehmung bei den Rezipient_innen. Verknüpft werden diese beiden Schritte mit dem Konzept des Agenda-Settings.
Dr. Regina Greck,

Diplom-Journalistin, ist Wissenschaftliche Mitarbeiterin am Studiengang Journalistik der Katholischen Universität EichstättIngolstadt. 


\section{Die Theorie des Agenda-Settings}

Die Theorie, dass die Medien mit ihrer Berichterstattung beeinflussen, worüber die Menschen nachdenken - wie bereits Cohen 1963 im Kernsatz des Agenda-Settings zusammenfasste -, wurde bereits mehrfach überprüft und weiterentwickelt (vgl. McCombs/Shaw 1972; Rössler 1997). Verschiedene Studien zeigen bis heute, dass dieser Effekt nachgewiesen werden kann - wenn auch in unterschiedlicher Stärke, abhängig von den untersuchten Themen, deren Aufmachung und der Mediengattung, in der sie erscheinen (vgl. Wanta/Ghanem 2007).

Die drei Grundbegriffe des Agenda-Setting-Konzeptes sind Thema, Tagesordnung sowie Themensetzung (vgl. dazu im Folgenden Maurer 2010, S. 10f.). Das Thema umfasst in der vorliegenden Studie die politisch aufgeladene Diskussion um die Olympischen Winterspiele in Sotschi. Die Tagesordnung bezeichnet die Rangfolge der Themen in der medialen Berichterstattung und beim Publikum. Diese Untersuchung befasst sich in einem engen Zuschnitt mit der Rangfolge verschiedener Aspekte innerhalb der medialen Diskussion um die Spiele in Russland 2014. Der Begriff der Themensetzung meint den Einfluss der Agenda der journalistischen Berichterstattung u.a. auf die des Publikums, die diese Studie vorsichtig nachzuzeichnen versucht.

Inwieweit die Agenda des Journalismus die des Publikums beeinflusst, beschreiben drei mögliche Modelle, die bereits McCombs 1977 (S. 99ff.) erforscht hat (vgl. zu den Modellen auch Rössler 1997, S. 93ff.; Maurer 2010, S. 16). Die Aufmerksamkeit der Rezipient_innen wird im (1) Awareness-Modell durch die journalistische Berichterstattung erst auf Themen gelenkt, während im (2) Salience-Modell die Häufigkeit der medialen Thematisierung die Wichtigkeit beim Publikum steigert. Im (3) Priorities-Modell übernehmen die Rezipient_innen die Agenda der Medien in der gleichen Rangfolge. Weitere Forscher entdeckten komplexere Mechanismen des Agenda-Settings, die die mediale Karriere von Themen und deren Auswirkung auf das Publikum im Fokus haben (vgl. Kepplinger et al. 1989, S. 99ff.), die Eigenschaften sowie die Mediennutzung der Rezipient_innen in den Blick nehmen (vgl. Erbring et al. 1980; Rössler 1997, S. 6of) und zudem zeigen, dass es auch zu einer Übernahme der Bewertung der Themen aus der journalistischen Berichterstattung durch das Publikum kommen kann (vgl. McCombs/Shaw 1993). Die vorliegende Studie zielt jedoch lediglich auf den Einfluss der journalistischen Themenagenda auf die Rezipient_innen ab. 


\section{Überregionale Berichterstattung und Publikumsmeinung in der Analyse}

Welche Themenaspekte rund um die Olympischen Spiele von Sotschi 2014 wie stark in den Medien beleuchtet wurden, untersucht diese Studie mittels einer quantitativen Inhaltsanalyse überregionaler Qualitätstageszeitungen („Süddeutsche Zeitung“ (SZ), „Frankfurter Allgemeine Zeitung“ (FAZ), „taz.die tageszeitung“ und „Die Welt“), ergänzt um die „Bild“-Zeitung sowie die politischen Nachrichtenmagazine „Spiegel“, „Stern“ und „Focus“. Der Untersuchungszeitraum umspannt ein halbes Jahr vor den Olympischen Winterspielen 2014 bis zu ihrem Ende (23.8.2013 bis 23.2.2014). Als Aufgreifkriterium für die analysierten Artikel mussten diese die Schlagworte ${ }^{1}$ „Sotschi“ oder „Sotchi“ sowie „Olympia“ und mindestens einen politischen Aspekt rund um die Spiele im ersten Absatz aufweisen. Insgesamt konnten 354 Artikel gefunden und untersucht werden.

Um diese inhaltsanalytisch ermittelte Themenagenda mit der des Publikums zu vergleichen, wurde die deutsche Bevölkerung zu ihrer Themenwahrnehmung sowie zu ihrer Einstellung zu Olympia in Sotschi befragt. ${ }^{2}$ Die Feldphase der Onlinebefragung im Pull-Verfahren erstreckte sich vom 26. Mai bis 8. Juni 2014 und ergab einen Rücklauf von 750 ausgefüllten Fragebögen. Der Link zum Fragebogen wurde in Internetforen und auf Facebook-Seiten publiziert, die sich um die Winterspiele in Sotschi, aber auch Olympia und Sportthemen allgemein drehen. Weiterhin unterstützten verschiedene Sport- und Journalistenverbände deutschlandweit die Studie und schickten die Befra-

1 Für die Übertragung des Austragungsortes aus dem Kyrillischen ins Lateinische gibt es folgende Möglichkeiten: Sotschi und Sotchi. Obwohl Sotschi die gängigere Schreibweise im Deutschen ist, tauchten beide in der journalistischen Berichterstattung auf und wurden demnach als Schlagwort verwendet.

2 Die vorliegende Studie wurde im Rahmen eines Lehrprojekts im Sommersemester 2014 an der Katholischen Universität Eichstätt-Ingolstadt unter der Leitung von Tanja Kössler und Dr. Regina Greck durchgeführt. Aufgrund der zeitlichen Determinanten des Semesters fand die Befragung der Rezipient_innen ca. drei Monate nach den Olympischen Spielen sowie nur einmalig und zeitgleich zur Inhaltsanalyse statt. Dieses Vorgehen ist nicht optimal, um Agenda-Setting-Effekte messen zu können, es kann sie nur andeuten. Zudem fokussiert die vorliegende Studie aus forschungsökonomischen Gründen nur Printmedien, wodurch die Live-Berichterstattung des Fernsehens, das ebenfalls Einfluss auf das Agenda-Setting beim Publikum hat, außen vor blieb. 
gung an ihre Mitglieder per Mail. Im ersten Schritt soll im Folgenden die politische Berichterstattung über die Olympischen Spiele von Sotschi 2014 in den ausgewählten überregionalen Medien betrachtet werden, um diese im zweiten Schritt mit der Wahrnehmung bei den Rezipient_innen zu vergleichen.

\section{Merkmale der Berichterstattung rund um Olympia in Sotschi}

Im Zeitverlauf wird deutlich, dass zu Beginn des Untersuchungszeitraums kaum politische Artikel in der Berichterstattung erscheinen (August bis November 2013: 16,1 Prozent, $\mathrm{n}=354$ ), während der Medienoutput kurz vor dem Beginn der Spiele stark zunimmt: Im Dezember 2013 werden 20,9 Prozent aller Artikel veröffentlicht, im Januar 2014 16,9 Prozent und im Februar 2014 46,o Prozent. Vor Beginn der Spiele, also bis zum 7. Februar 2014, berichten die untersuchten Printmedien mit im Schnitt neun Artikeln politischer Natur pro Woche, während der Wettkämpfe erhöhte sich die Zahl auf 67.

Ein knappes Drittel der Analyseeinheiten (30,8 Prozent) waren Meldungen, 32,5 Prozent Berichte. Andere Darstellungsformen wie Interviews (5,9 Prozent) und Reportagen (6,5 Prozent) wählen die Medien im Zusammenhang mit Olympia in Sotschi deutlich seltener. Mit einem Fünftel (20,6 Prozent) ist der Anteil der Kommentare jedoch ziemlich groß. Das meinungsgeladene Thema scheint demnach die Form der Berichterstattung beeinflusst zu haben.

\section{Themen in der journalistischen Berichterstattung}

Alle untersuchten Artikel wurden zunächst in thematische Bereiche sortiert und anschließend einem konkreten Unterthema zugeordnet. Mit Abstand am häufigsten behandeln die analysierten Artikel die Themenfelder Gesellschaftspolitik (29,9 Prozent) und die Ausrichtung der Olympischen Spiele (25,1 Prozent). Die gesellschaftspolitischen Artikel thematisieren u.a. den Umgang mit politischen Oppositionellen (33,0 Prozent), mit Homosexuellen (32,1 Prozent) sowie mit den Menschenrechten in Russland allgemein (12,3 Prozent).

Bei der Ausrichtung der Spiele werden vor allem die Aspekte Sicherheit (26,7 Prozent), Arbeitsbedingungen beim Bau der Sportstätten (23,3 Prozent), Kosten der Spiele (16,7 Prozent) und die Umweltproblematik rund um Sotschi (11,1 Prozent) zur Sprache gebracht. 
Die Reaktionen anderer Nationen auf Russland als Gastgeberland machen 13,6 Prozent aller Analyseeinheiten aus. Dabei diskutierten die Autoren dieser Artikel in rund zwei Dritteln der Fälle (66,7 Prozent) den Boykott von Olympia durch Staatschefs und Politiker, während nur 6,3 Prozent den expliziten Besuch von Staatsmännern in den Fokus rückten. Einen sportlichen Schwerpunkt hatten insgesamt 12,4 Prozent aller untersuchten Analyseeinheiten, während 4,8 Prozent die Trennung von Sport und Politik thematisierten und 2,3 Prozent positive Auswirkungen der Spiele auf Russland (11,9 Prozent Sonstiges).

\section{Themen im Zeitverlauf in der Tagespresse}

Anhand der sechs am häufigsten gefundenen Unterthemen (vgl. Abb. 1) wird deutlich, dass die politisch geprägte Berichterstattung in den analysierten Tageszeitungen hauptsächlich vor Beginn der Olympischen Spiele in Sotschi stattfindet, bei der „Bild“-Zeitung ausschließlich. Über den Boykott der Wettkämpfe u. a. von Bundespräsident Joachim Gauck schreiben die untersuchten Medien nur im Vorfeld, doch auch die Sicherheitsthematik und die Kosten der Spiele erzeugen nach der Eröffnungsfeier kaum noch ein Medienecho. Lediglich die Diskussion um das umstrittene Gesetz gegen positive Äußerungen über Homosexualität im Beisein Minderjähriger oder in den Medien bleibt in der Berichterstattung relativ konstant, noch deutlicher ist dies für Artikel über den Umgang mit Oppositionellen in Russland zu konstatieren.

\section{Personalisierungstendenz in der Berichterstattung}

Die Inhaltsanalyse ermöglichte es auch, die zwei prominentesten Akteure der Artikel festzuhalten. In rund 8o Prozent aller Fälle lässt sich ein politischer Akteur (dazu zählen Politiker oder Aktivisten) ausmachen, knapp 6o Prozent der Artikel nennen (auch) einen Akteur aus dem Sport wie Trainer, Athleten oder Vertreter von Verbänden (vgl. Tab. 1). Sowohl bei den Politikern als auch bei den Akteuren des Sports zeigt sich eine hohe Konzentration auf einzelne Personen. In 15,1 Prozent der Artikel steht dezidiert das Olympische Komitee und/oder sein Präsident Thomas Bach (12,2 Prozent) im Fokus. Als politische Akteure treten der russische Präsident Wladimir Putin (25,2 Prozent) und/oder der deutsche Bundespräsident Joachim Gauck (8,4 Prozent) am häufigsten in den Artikeln auf. 


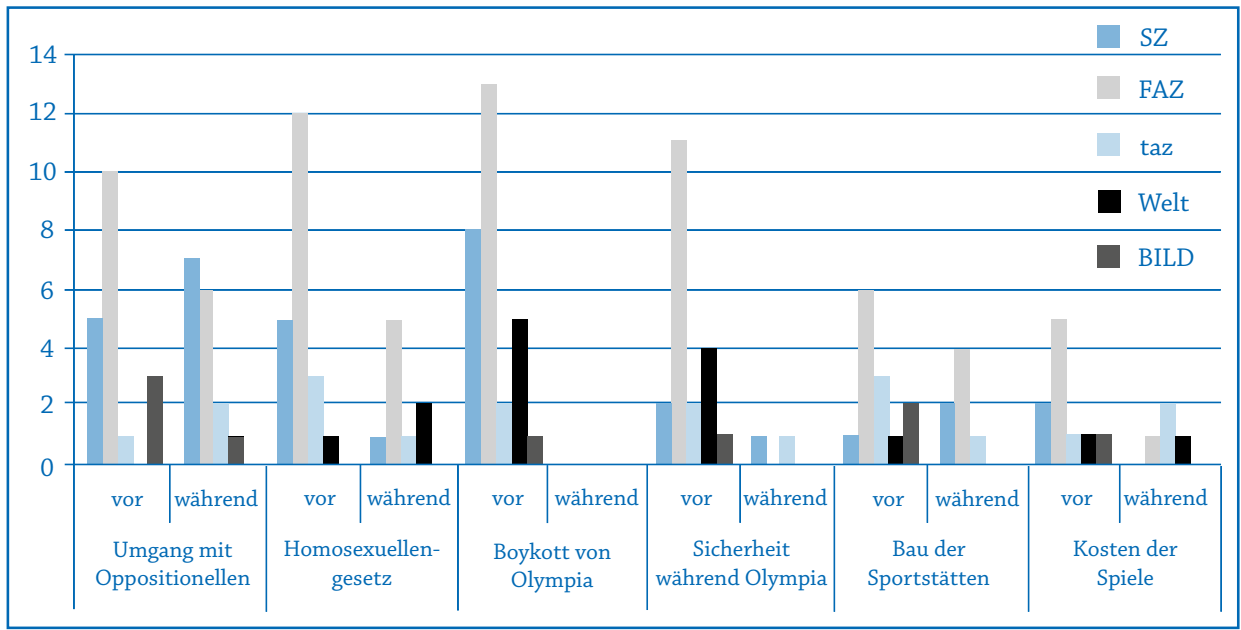

Abb.1: Die häufigsten sechs Themen in der Berichterstattung rund um die Olympischen Spiele von Sotschi 2014 nach Medium sowie vor und während der Spiele ( $n=149$, absolute Zahlen)

Zusammenfassend zeigt sich, dass in der Berichterstattung rund um die Olympischen Winterspiele von Sotschi 2014 gesellschaftspolitische Themen und solche, die die Ausrichtung der Spiele betreffen, in der überregionalen Presse im Vordergrund stehen. Welche Aspekte die Rezipient_innen wahrgenommen haben, zeigt im Folgenden die Auswertung der quantitativen Befragung.

\section{Eingeschränktes Befragungssample}

Mit 750 gültigen Antworten erreichte die Umfrage nach der Datenbereinigung eine Beendigungsquote von 53,4 Prozent. Wie bei einem Lehrprojekt zu erwarten war, weist die Stichprobe einige Besonderheiten auf. Während im Geschlechterverhältnis (48,7 Prozent Männer und 51,3 Prozent Frauen) keine großen Differenzen zum gesamtdeutschen Durchschnitt auszumachen sind (vgl. Statistisches Bundesamt 2015), macht der Altersdurchschnitt der Befragten von rund 35 Jahren deutlich, dass es sich bei den Teilnehmern der Umfrage um ein eher jüngeres Publikum handelt, das unter dem Vergleichswert der deutschen Bevölkerung liegt (Altersmedian Deutschland 2015: 46,2 Jahre; vgl. UN DESA (Population Division) 2015). Noch deutlicher weist das Bildungsniveau der Befragten auf ein stark universitär geprägtes Milieu der Teilnehmer hin. So geben 8o,2 Prozent an, mindestens das Abitur abgelegt zu haben, knapp über die Hälfte der Abiturient_innen (59,1 Prozent) verfügt darüber hinaus mindestens über ein abgeschlossenes Studium. Obwohl 


\begin{tabular}{|l|c|}
\hline Akteur & Prozent der Artikel \\
\hline Verbände & $34,8 \%$ \\
\hline Sportler \& Trainer & $23,5 \%$ \\
\hline Organisationen \& Aktivisten & $7,8 \%$ \\
\hline russische Organisationen \& Aktivisten & $15,1 \%$ \\
\hline deutsche Politiker & $18,0 \%$ \\
\hline russische Politiker & $33,9 \%$ \\
\hline sonstige Politiker & $7,2 \%$ \\
\hline sonstige Akteure & $29,3 \%$ \\
\hline
\end{tabular}

Tab. 1: Akteure in der Berichterstattung rund um die Olympischen Spiele von Sotschi 2014 (die häufigsten Akteure, Prozent der Artikel)

die Teilnehmer der Befragung online und deutschlandweit rekrutiert wurden, ergibt sich ein deutliches regionales Muster, was die Herkunft der Teilnehmer angeht. Mehr als drei Viertel aller Befragten (78,3 Prozent) kommen aus Bayern, weitere 8,4 Prozent antworteten aus Baden-Württemberg. Die restlichen Bundesländer stellen nur marginal Befragungsteilnehmer.

\section{Sport und Politik}

\section{- verschwimmende Grenzen?}

Bei der Austragung der Olympischen Spiele geht es schon lange nicht mehr nur um die Wettkämpfe vor Ort. In Zeiten der Medialisierung der Gesellschaft (vgl. Meyen 2009, S. 23; Jarren/ Donges 2011, S. 24; Schulz 2011, S. 31ff.) können solche Großereignisse unterschiedliche Interessensgruppen aus Politik, Wirtschaft und natürlich dem Sport auf den Plan rufen, die dort eine mediale Plattform suchen. Die Befragten sollten ihre Einschätzung zu diesen verschwimmenden Grenzen in der Bewertung verschiedener Aussagen auf einer vierstufigen Skala ${ }^{3}$ abgeben. Der Großteil der Teilnehmer (61,2 Prozent) stimmt sehr oder teils dem Statement zu, dass eigentlich die sportlichen Wettkämpfe rund um die Olympischen Spiele im Fokus stehen sollten. Trotzdem glaubt ebenfalls die Mehrheit (62,6 Prozent) sehr oder teils, dass Olympia durchaus auch eine ge- 
eignete Plattform für politische Aussagen ist. 79,6 Prozent der Teilnehmer hätten sich in Bezug auf Olympia in Sotschi sogar sehr oder teils gewünscht, dass das Olympische Komitee Stellung zu politischen Fragen bezogen hätte. Auch die wirtschaftlichen Interessen überlagern dem Eindruck von fast der Hälfte der Befragten nach die sportlichen: 45,6 Prozent der Teilnehmer attestieren diesen sehr oder teilweise eine höhere Relevanz im Rahmen der Olympischen Spiele als den Wettkämpfen.

\section{Themenwahrnehmung des Publikums}

Die Befragten sollten im Fragebogen insbesondere beurteilen, ob und in welchem Maß sie bestimmte Themenfelder in der journalistischen Berichterstattung wahrgenommen haben. Dabei fällt zunächst ins Auge, dass nur 29,2 Prozent der Teilnehmer die Politik im Vergleich zum Sport im Vordergrund sehen, wohingegen der Rest das Verhältnis andersherum oder zumindest als ausgeglichen empfindet. Gefragt nach der Beurteilung der politischen Lage in Russland durch die journalistische Berichterstattung der deutschen Medien geben die meisten Teilnehmer an (82,4 Prozent), diese auf einer fünfstufigen Skala ${ }^{4}$ als mindestens zurückhaltend kritisch erlebt zu haben. Den Befragten wurden anschließend acht konkrete Themenfelder präsentiert, die sich in einer qualitativen Vorstudie von Teilen des Materials der quantitativen Inhaltsanalyse als die prominentesten in der Medienberichterstattung ergeben haben. Die Umfrageteilnehmer sollten die Präsenz dieser Themen in den Medien auf einer vierstufigen Skala von sehr häufig bis nie bewerten.

Am deutlichsten erinnerten sich die Befragten an Artikel über den Umgang mit Homosexuellen in den deutschen $\mathrm{Me}$ dien (vgl. Tab. 2). Rund drei Viertel (77,6 Prozent) gaben an, dass das oft bis sehr häufig Thema in den Medien war. Ähnliche Angaben erzielte die Berichterstattung über den Umgang der russischen Politik mit Oppositionellen (78,7 Prozent sehr häufig und oft), wobei die Befragten dabei seltener als beim Thema Homophobie sehr häufig angaben. Nach diesen beiden Spitzenthemen folgen in der Erinnerung der Befragten in der Berichterstattung um die Olympischen Spiele in Sotschi ebenfalls oft die immensen Kosten der Ausrichtung (70,1 Prozent), die prekären Arbeitsbedingungen auf den Baustellen (65,5

4 Die Skala lautete: kritisch, zurückhaltend kritisch, neutral, eher unkritisch, unkritisch. 
Prozent) und die Klimaproblematik, die sich im Badeort Sotschi ergab, als er zu einem Wintersportort umgestaltet werden musste (61,7 Prozent).

Selten bis nie fanden nach Ansicht der Mehrheit der Befragten die Umsiedlung von Einheimischen (59,2 Prozent), polizeiliche Eingriffe bei der Kartenvergabe (86,6 Prozent) und die problematische Abfallentsorgung auf dem Areal der Spiele (88,2 Prozent) in den Medien statt.

\section{Vergleich von Publikums- und Medienagenda}

Der Vergleich dieser Angaben mit den Ergebnissen der Inhaltsanalyse zeigt einige interessante Überschneidungen. Es wird deutlich, dass die am häufigsten durch die Befragten wahrgenommenen Themen auch die am häufigsten in den überregionalen Zeitungen und Nachrichtenmagazinen publizierten sind (vgl. Tab 3). ${ }^{5}$

So findet sich der Umgang mit politischen Oppositionellen und mit Homosexuellen jeweils auf Platz 1 und 2 beider Themenranglisten. Auch die Kosten der Ausrichtung der Spiele und die schlechten Arbeitsbedingungen auf den Baustellen bekommen einen ähnlichen Rangplatz auf beiden Listen, bevor diese sich in der Themenhäufigkeit auseinander bewegen.

Einschränkend gilt anzumerken, dass die quantitative Inhaltsanalyse dieser Studie, über die die Themenrangliste der Medien erstellt wurde, nur auf überregionalen Printmedien basiert. Andere Mediengattungen blieben aus forschungsökonomischen Gründen außen vor, obwohl Fernsehen, Internet und Radio natürlich auch Einfluss auf die Rezipient_innen haben können. Eine Kotrollfrage in der Befragung zeigt, dass das Fernsehen bei den Teilnehmern als das häufigste Medium gewählt wurde, um sich über Olympia in Sotschi zu informieren: Rund die Hälfte aller Befragten nutzte es sehr häufig oder oft dafür. Dies entspricht in etwa der Mediennutzung der Deutschen im Durchschnitt (vgl. Media Perspektiven 2014, S. 66) und ist im vorliegenden Fall sicher auch dem geschuldet, dass der Live-Charakter des TV ein sportliches Thema am besten wiedergibt. An zweiter Stelle der Informationsquellen der Befragten über Olympia in

5 Aufgrund der methodischen Einschränkungen durch den zeitlichen Rahmen eines Lehrprojektes, die bereits in Fußnote 2 angedeutet wurden, können nur Ranglisten der Themen in Befragung und Inhaltsanalyse verglichen werden. 


\begin{tabular}{|l|c|c|}
\hline $\begin{array}{l}\text { Themen in Bezug auf Olympia } \\
\text { (Angaben in Prozent) }\end{array}$ & sehr häufig/oft & selten/nie \\
\hline Umgang mit politischen Oppositionellen & 78,7 & 21,3 \\
\hline Umgang mit Homosexuellen & 77,7 & 22,3 \\
\hline Kosten für die Ausrichtung der Spiele & 70,1 & 29,9 \\
\hline Arbeitsbedingungen auf den Baustellen & 65,5 & 34,5 \\
\hline $\begin{array}{l}\text { Umgestaltung eines Badeortes } \\
\text { in einen Wintersportort }\end{array}$ & 61,7 & 38,3 \\
\hline Umsiedlung von Einheimischen & 40,8 & 59,2 \\
\hline $\begin{array}{l}\text { Polizeiliche Eingriffe bei der Karten- } \\
\text { und Passvergabe }\end{array}$ & 13,4 & 86,6 \\
\hline Probleme bei der Abfallentsorgung & 11,8 & 88,2 \\
\hline
\end{tabular}

Tab. 2: Die Wahrnehmung politischer Problemfelder rund um die Spiele in den Medien durch die Befragten $(n=743-746)$

Sotschi folgen schon die Zeitung (36,2 Prozent) und das Internet (34,5 Prozent). Eher wenig genutzt wurde das Radio. Gerade einmal rund ein Fünftel der Befragten hörte oft oder sehr häufig Informationen zu den Olympischen Spielen.

Die gefundene grobe Übereinstimmung der Themenagenden des Publikums und der analysierten Printmedien zeigt, dass beide offensichtlich nicht losgelöst voneinander existieren. Die Ergebnisse der vorliegenden Studie können vermuten lassen, dass die Medien in Anlehnung an Cohen (1963) und den Grundgedanken des Agenda-Settings beeinflussen können, was die Rezipient_innen zu einem Thema wahrnehmen. Ob sie das „Wie“ beeinflussen können, wie McCombs und Shaw (1993) vermuten, bliebe für die Thematik der Olympischen Spiele in Sotschi noch zu erforschen.

\section{Literatur}

Cohen, Bernard Cecil (1963): The press and foreign policy. Princeton.

Erbring, Lutz/Goldenberg, Edie N./Miller, Arthur (1980): Front-Page News and Real World Cues: A New Look at Agenda-Setting by Mass Media. In: American Journal of Political Science, 24. Jg., Nr. 4, S. 383-395.

Jarren, Otfried/Donges, Patrick (32011): Politische Kommunikation in der Mediengesellschaft. Eine Einführung. Wiesbaden. 


\begin{tabular}{|l|c|c|}
\hline Themen in Bezug auf Olympia & $\begin{array}{c}\text { Rangplatz Befragung } \\
\text { sehr häufig/oft } \\
\text { in den Medien } \\
\text { wahrgenommen }\end{array}$ & $\begin{array}{c}\text { Rangplatz } \\
\text { Inhaltsanalyse } \\
\text { Häufigkeit gefundene } \\
\text { Themen }\end{array}$ \\
\hline Umgang mit politischen Oppositionellen & 1 & 1 \\
\hline Umgang mit Homosexuellen & 2 & 2 \\
\hline Kosten für die Ausrichtung der Spiele & 3 & 6 \\
\hline Arbeitsbedingungen auf den Baustellen & 4 & 5 \\
\hline Umgestaltung eines Badeortes & 5 & 10 \\
\hline in einen Wintersportort & 6 & 13 \\
\hline Umsiedlung von Einheimischen & 7 & 18 \\
\hline Probleme bei der Abfallentsorgung & & 5 \\
\hline
\end{tabular}

Tab. 3: Rangliste der am häufigsten durch die Befragten wahrgenommenen Themen in der journalistischen Berichterstattung und ihr Rangplatz in der durch die in der Inhaltsanalyse erstellten Themenliste nach Häufigkeit

Kepplinger, Hans Mathias et al. (1989): Der Einfluss der Fernsehnachrichten auf die politische Meinungsbildung. Freiburg im Breisgau.

Maurer, Marcus (2010): Agenda-Setting. Baden-Baden.

McCombs, Maxwell E./Shaw Donald L. (1972): The Agenda-Setting Function of Mass Media. In: The Public Opinion Quarterly, 36. Jg., Nr. 2, S. 176-187.

McCombs, Maxwell E. (1977): Newspapers Versus Television: Mass Communication Effects Across Time. In: Shaw, Donald L./McCombs, Maxwell E. (Hg.): The emergence of American political issues. The Agenda-Setting Function of the Press. St. Paul, S. 89-106.

McCombs, Maxwell E./Shaw, Donald L. (1993): The Evolution of Agenda-Setting Research: Twenty-Five Years in the Marketplace of Ideas. In: Journal of Communication, 43. Jg., Nr. 2, S. 58-67.

Media Perspektiven (2014): Basisdaten. Frankfurt am Main.

Meyen, Michael (2009): Medialisierung. In: Medien \& Kommunikationswissenschaft, 57. Jg., Nr. 1, S. 23-38.

Rössler, Patrick (1997): Agenda-Setting. Theoretische Annahmen und empirische Evidenzen einer Medienwirkungshypothese. Opladen.

Schulz, Winfried (32011): Politische Kommunikation: Theoretische Ansätze und Ergebnisse empirischer Forschung. Wiesbaden.

Statistisches Bundesamt 2015: Bevölkerung - Zahl der Einwohner in Deutschland nach Geschlecht von 1995 bis 2014. Zugriff über Statista. http:// de.statista.com/statistik/daten/studie/161868/umfrage/entwicklung-der- 
gesamtbevoelkerung-nach-geschlecht-seit-1995/ (zuletzt aufgerufen am 30.5.2016).

UN DESA (Population Division) 2015: Durchschnittsalter der Bevölkerung in den wichtigsten Industrie- und Schwellenländern im Jahr 2015 (Altersmedian in Jahren). Zugriff über Statista. http://de.statista.com/statistik/ daten/studie/37220/umfrage/altersmedian-der-bevoelkerung-in-ausgewaehlten-laendern/ (zuletzt aufgerufen am 30.5.2016).

Wanta, Wayne/Ghanem, Salma (2007): Effects of agenda-setting. In: Preiss, Raymond W. et al. (Hg.): Mass media effects research. Advances through media-analysis. New York, S. 37-52. 